\title{
Structure-function correlations in copper clusters in proteins
}

\author{
Edward I. Solomon, James L. Cole, and Michael J. Baldwin \\ Department of Chemistry, Stanford University, Stanford, CA 94305, USA
}

Abstract- A coupled binuclear copper active site is present in a wide variety of proteins and enzymes which perform different biological functions utilizing $\mathrm{O}_{2}$. In the multicopper oxidases, the Type 2 and Type 3 centers comprise a trinuclear $\mathrm{Cu}$ cluster which represents the active site for the multielectron reduction of $\mathrm{O}_{2}$.

A coupled binuclear copper active site is present in a wide variety of proteins and enzymes which perform different biological functions utilizing $\mathrm{O}_{2}$. The hemocyanins (Hc) reversibly bind $\mathrm{O}_{2}$, the tyrosinases (Ty) are monoxygenases which hydroxylate monophenols to o-diphenols and oxidize these to o-quinones, and the multicopper oxidases (laccase (Lc), ascorbate oxidase and ceruloplasmin), which contain additional copper centers (Type 1 and Type 2) catalyze the four electron reduction of $\mathrm{O}_{2}$ to water. Our original chemical and spectroscopic studies over a series of protein active site derivatives of $\mathrm{Hc}$ and $\mathrm{Ty}$ demonstrated (ref. 1,2) that these proteins have very similar active sites. The key features of this spectroscopically effective model for the coupled binuclear copper active site, reproduced in Fig. 1 , are that dioxygen binds as peroxide, bridging two tetragonal $\mathrm{Cu}$ (II)'s in a cis $\mu-1,2$ fashion, with an additional endogenous bridge between the coppers (RO'). The endogenous bridge is likely hydroxide based on the crystal structure (ref. 3 ) of deoxy Hc. The major difference between the $\mathrm{Hc}$ and Ty sites is the high accessibility of the Ty active site to exogenous ligands (ref. 4). We found that substrate analogues bind directly to the copper in Ty and compete with peroxide for the same binding site (ref. 5). These studies resulted in the proposed structural mechanism for hydroxylation and oxidation catalysis given in Fig. 2. Alternatively, chemical and spectroscopic studies of a series of protein active site derivatives of $\mathrm{Lc}$ (which is the simplest multicopper oxidase, containing one Type 1, one Type 2, and one coupled binuclear Type 3 center) showed the Type 3 site to be strikingly different from the coupled binuclear site in Hc and Ty (ref. 6). Low-temperature magnetic circular dichroism (LTMCD) spectroscopy was found to be a powerful probe of the different copper centers in the multicopper oxidases, allowing a correlation of excited state spectral features with the ground state magnetic properties. From LTMCD studies, the Type 3 site was in fact found to be part of a trinuclear copper cluster with the Type 2 center (ref. 7). This new class of copper site has recently been supported by

crystallographic studies on ascorbate oxidase (ref. 8). Our recent studies have focussed on I) Spectroscopy of model complexes to further develop our understanding of the unique spectral features of oxy Hc; II) A detailed comparison of the coupled binuclear site in Hc and the Type 3 site in Lc; III) Evaluation of the metal centers required for the $\mathrm{O}_{2}$ reactivity of the multicopper oxidases; and IV) Detailed LTMCD spectral studies of the trinuclear copper cluster to establish its geometric and electronic structure and interactions with exogenous ligands as related to the mechanism of multielectron reduction of $\mathrm{O}_{2}$. These studies are summarized below.

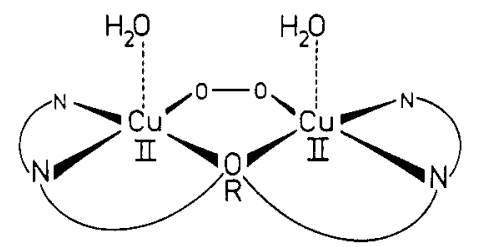

Fig. 1. The Spectroscopically Effective Active Site of Hemocyanin and Tyrosinase.

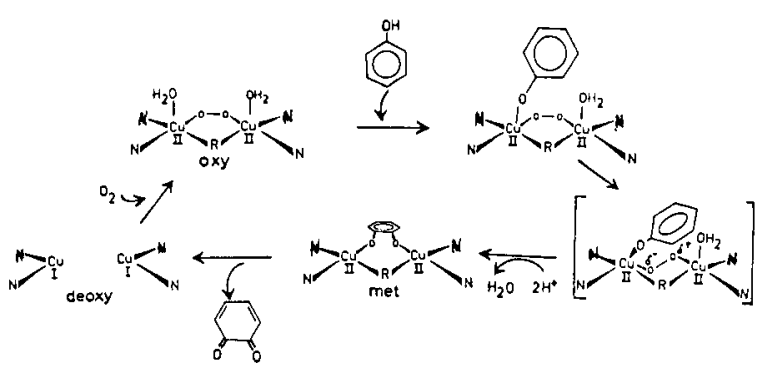

Fig. 2. Active site structural mechanism for hydroxlyation and oxidation of phenols to form o-quinones by tyrosinase.

\section{ELECTRONIC STRUCTURE OF OXY HEMOCYANIN}

In oxy Hc, the two $\mathrm{Cu}$ (II) ions show strong antiferromagnetic coupling in the ground state, and are consequently inaccessible to ground state spectral study. The excited state spectroscopic features are therefore the most useful probe of the electronic structure of 
the oxy Hc active site. By comparison of oxy Hc to met aquo Hc, a fully oxidized derivative of $\mathrm{Hc}$ in which peroxide has been displaced, the intense oxy Hc absorption (abs.) bands at $345 \mathrm{~nm}\left(\varepsilon=20,000 \mathrm{M}^{-1} \mathrm{~cm}^{-1}\right)$ and at $570 \mathrm{~nm}\left(\epsilon=1000 \mathrm{M}^{-1} \mathrm{~cm}^{-1}\right)$, as well as the CD band at $480 \mathrm{~nm}\left(\Delta \epsilon-+2.5 \mathrm{M}^{-1} \mathrm{~cm}^{-1}\right.$ ), have been assigned as $0_{2}{ }^{2-} \rightarrow \mathrm{Cu}$ (II) charge transfer (CT) transitions (ref. 1). In order to gain insight into the electronic structure of oxy Hc, a transition dipole vector coupling (TDVC) model has been developed for analysis of CT transitions of exogenous bridging ligands and has been tested on several small molecule model complexes. The highest occupied molecular orbitals (HOMOs) of peroxide are a degenerate $\pi^{*}$ set which splits in energy upon coordination to copper, with one $\sigma$ bonding to the half-filled $d\left(x^{2}-y^{2}\right)$ acceptor orbital of the copper. The CT transition from the former $\left(\pi_{\sigma}^{*}\right)$ will be higher in energy and more intense, due to greater overlap, than that from the latter $\left(\pi_{\mathrm{v}}^{*}\right)$. Peroxide has been shown by mixed-isotope resonance Raman studies to bind terminally to one copper in a binuclear copper complex prepared by Karlin et al. (ref. 9). The $r R$ enhancement profiles of the (0-0) and ( $\mathrm{Cu}-0)$ stretching modes show two $\mathrm{O}_{2}{ }^{2-} \rightarrow \mathrm{Cu}$ (II) CT transitions, with the more intense feature at higher energy (505nm, $\epsilon=2400 \mathrm{M}^{-1} \mathrm{~cm}^{-1}$; $610 \mathrm{~nm}, \epsilon-2400 \mathrm{M}^{-1} \mathrm{~cm}^{-1}$ ) as predicted above.

The HOMOs of azide are a $\pi^{\text {nb }}$ degenerate set, similar to the $\pi^{*}$ set in peroxide. Copper complexes with azide terminally bound show one $\mathrm{N}_{3}{ }^{\circ} \rightarrow \mathrm{Cu}$ (II) CT transition in the absorption spectrum at $400 \mathrm{~nm}\left(\epsilon=2000 \mathrm{M}^{-1} \mathrm{~cm}^{-1}\right)$, indicating that only the $\pi_{\sigma}^{\mathrm{nb}} \rightarrow$ Cu transition contributes significant intensity. In the $\mu-1,3$ azide bridged copper dimers, two $\mathrm{N}_{3} \rightarrow \mathrm{Cu}$ (II) CT transitions are seen at $365 \mathrm{~nm}\left(\epsilon-2000 \mathrm{M}^{-1} \mathrm{~cm}^{-1}\right)$ and $420 \mathrm{~nm}\left(\epsilon=1000 \mathrm{M}^{-1} \mathrm{~cm}^{-1}\right)$. From the TDVC model, these are the symmetric and antisymmetric combinations of the $\pi_{\sigma}^{\text {nb } \rightarrow C u}$ (II) transition in the monomer, both of which are electric dipole allowed. The energy spitting estimated from the Coulomb interactions between the transition moments is significantly less than the experimental value, which has been accounted for by introduction of an exchange mediated excitation transfer contribution (the $\mathrm{L}$ integral) to the excited state energies. The met azide derivative of Hc shows spectroscopic features which are remarkably similar to those of Reed's $\mu-1,3$ azide bridged dimer model, demonstrating the applicability of the TDVC model to the spectra of the protein site (ref. 10).

The abs. and CD spectra of oxy Hc, shown in Fig. 3, are interpreted within the framework of this TDVC model (ref 1. ). In the $\mu-1,2$ peroxide bridged structure in Fig. 1 , each of the

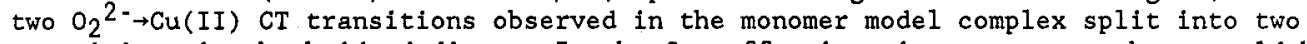
transitions in the bridged dimer. In the $C_{2} v$ effective site symmetry, these would be of $A_{1}$ and $B_{1}$ symmetry for $\pi_{\sigma}^{*}$ and $A_{2}$ and $B_{2}$ for $\pi_{v}^{*}$. All except the $A_{2}$ transition should be electric dipole allowed, while the $A_{2}, B_{2}$, and $B_{1}$ transitions should be magnetic dipole allowed. Thus, $\triangle A / A$ must be greatest for the $A_{2}$ transition, and the $480 \mathrm{~nm} C D$ band can be assigned to this transition. The $570 \mathrm{~nm}$ abs. band is assigned as the electric dipole allowed $B_{2}$ component of $\pi_{\mathrm{v}}^{*}$. This ordering of the $A_{2}$ and $B_{2}$ transitions is as calculated using the Coulomb interactions, but as in the azide complexes the splitting is underestimated $\left(840 \mathrm{~cm}^{-1}\right.$ compared to the observed value of $3500 \mathrm{~cm}^{-1}$ ). For the $\pi_{\sigma}^{*}$ transitions, $B_{1}$ is expected to be at higher energy than $A_{1}\left(E\left(A_{1}\right)-E\left(B_{1}\right)\right.$ is calculated to be $-5000 \mathrm{~cm}^{-1}$ ), with $A_{1}$ more intense based on vector coupling of the CT moments. Thus, the $345 \mathrm{~nm}$ band is assigned as the $A_{1}$ component of $\pi_{\sigma}^{*}$. The fact that three $\mathrm{O}_{2}^{2-} \rightarrow \mathrm{Cu}$ (II) CT transitions are observed for oxy Hc requires that the peroxide bridges the active site, and these CT spectral features are shown to be well interpreted in the context of the TDVC model which has now been calibrated by structurally defined binuclear copper model complexes.

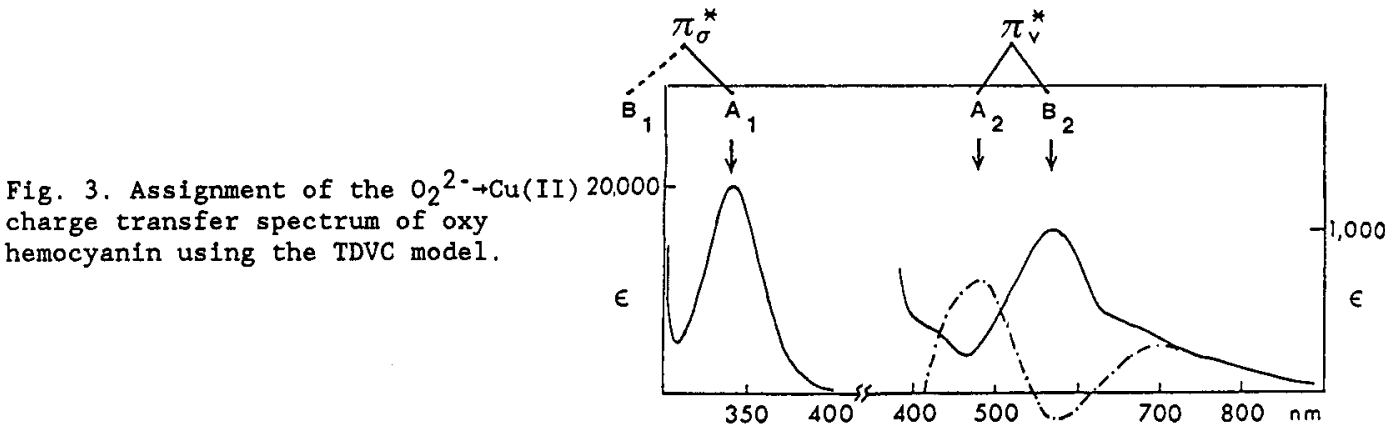

\section{COMPARISON OF THE BINUCLEAR SITE IN HC AND THE TYPE 3 SITE IN T2D LC}

A parallel series of met [Cu(II) $\mathrm{Cu}(\mathrm{II})]$, half met $[\mathrm{Cu}(\mathrm{I}) \mathrm{Cu}(\mathrm{II})]$ and deoxy [Cu(I) $\mathrm{Cu}(\mathrm{I})]$ derivatives of Hc and of the Type 3 site in a Type 2 depleted (T2D) derivative of Lc (where the Type $2 \mathrm{Cu}$ is reversibly removed and the Type $1 \mathrm{Cu}$ is oxidized) have been prepared. In the met form, both the Hc and Lc binuclear sites contain two tetragonal $\mathrm{Cu}$ (II) ions. However neither site gives rise to an EPR signal; therefore, the $\mathrm{Cu}(\mathrm{II})$ ions are antiferromagnetically coupled. Preliminary SQUID susceptibility measurements place a lower limit on the singlet-triplet splitting of $-2 \mathrm{~J}>400 \mathrm{~cm}^{-1}$ for met Hc and $-2 \mathrm{~J}>200 \mathrm{~cm}^{-1}$ for met T2D Lc. Lowering the $\mathrm{pH}$ and adding azide produces broad triplet EPR signals in both 
systems which account for $<108$ of the sites. These signals arise from two dipolar interacting $\mathrm{Cu}$ (II) ions. These results are interpreted in terms of an endogenous bridge in both met aquo sites which provides the superexchange pathway for strong antiferromagnetic coupling. Protonation of this bridge uncouples the sites, and from the $\mathrm{pH}$ dependence of the dipolar EPR signals the endogenous bridge ligands are calculated to have similar intrinsic $\mathrm{pk}_{\mathrm{a}}$ 's $(>6.7)$.

The spectroscopic and chemical properties of the half met Hc and T2D Lc sites are very different. In Hc, the electronic spectral features of the half met $\mathrm{NO}_{2}{ }^{-}$derivative indicate a localized $\mathrm{Cu}$ (II) center with - tetragonal geometry. However, an additional low energy band at $-1000 \mathrm{~nm}$ is observed in the $\mathrm{Br}^{-}$derivative. This band is reasonably intense in abs. but has very little associated LTMCD intensity. This allows assignment as an intervalent transfer (IT) transition, since the selection rules for C term MCD require two perpendicular transition moments and an IT transition is polarized only along the Cu-Cu axis. Detailed analysis of the IT transition over the half met- $\mathrm{L}$ series ( $\mathrm{L}-\mathrm{Cl}^{-}, \mathrm{Br}^{-}, \mathrm{I}^{-}$) allows an estimation of the electron delocalization between the coppers in the ground state wavefunction. The delocalization increases with increasing covalency of the exogenous ligand, indicating that this is the pathway for electron delocalization and thus must bridge the two coppers at the half met hc site (ref. 11).

Exogenous ligands bind to the half met Hc site with unusually high affinity (at $298 \mathrm{~K}$ : $\mathrm{K}_{\mathrm{N}_{3}}$ (half met) $-3.2 \times 10^{4}$, (met) -80 , (deoxy) $-150 \mathrm{M}^{-1}$ ). Temperature dependent studies indicate that this high affinity resuits from a very favorable entropy contribution. In contrast to half met $\mathrm{Hc}$, the spectral features of the mixed valent T3 site in half met T2D Lc show no evidence for electron delocalization. Furthermore, exogenous ligands bind to the mixed valent T3 site with affinities similar to aqueous $\mathrm{Cu}$ (II). Taken together, these results strongly suggest that exogenous ligands do not bridge two coppers at the half met Type 3 site in T2D LC (ref. 6).

Deoxy Hc contains a pair of $\mathrm{Cu}(\mathrm{I})$ ions and readily reacts with $\mathrm{O}_{2}$ to form oxy $\mathrm{Hc}$, which contains a pair of $\mathrm{Cu}$ (II) lons bridged by peroxide (Fig. 1). Using a quantitative Cu X-ray absorption edge method we developed to assay $\mathrm{Cu}$ redox state composition, we have demonstrated that T2D Lc as isolated contains an oxidized Type 1 site and a reduced Type 3 site (deoxy T2D) (ref. 12). In contrast to deoxy He the reduced Type 3 site in deoxy T2D LC is stable to oxidation by $\mathrm{O}_{2}$. The linear correlation of the formation of a $330 \mathrm{~nm}$ abs. band with the percent oxidation of the Type 3 site during peroxide titration of T2D Lc indicates that peroxide oxidizes but does not bind to the Type 3 site, thus casting doubt on the existence of peroxide intermediates in Lc. Like Hc, exogenous ligands bind at the deoxy T2D Lc active site, indicating that the lack of $\mathrm{O}_{2}$ reactivity in deoxy T2D Lc compared to deoxy $\mathrm{Hc}$ is not due to inaccessibility of small molecules, but likely relates to terminal compared to bridged exogenous ligand binding modes, respectively.

\section{III. $\mathrm{O}_{2}$ REACTIVITY OF LACCASE}

The reactivity of fully reduced $\mathrm{T} 2 \mathrm{D}$ and $\mathrm{T} 1 \mathrm{Hg} \mathrm{Lc}$ (a derivative in which the Type $1 \mathrm{Cu}$ is replaced by $\mathrm{Hg}$ (II), containing a Type 2-Type 3 trinuclear $\mathrm{Cu}$ cluster) with $\mathrm{O}_{2}$ has been investigated using $\mathrm{Cu} X$-ray absorption edge spectroscopy. Upon exposure of reduced T2D Lc to air, biphasic reoxidation kinetics are observed. A small amount of Cu reoxidizes quickly, which corresponds to $-10 \%$ native Lc regenerated under reducing conditions As monitored optically, the bulk of the Type 1 centers reoxidize slowly (half-time $-12 \mathrm{~h}$ ). However, an intense $\mathrm{Cu}(\mathrm{I})$ peak at $8984 \mathrm{eV}$ is present in the $\mathrm{Cu}$ edge of a sample exposed to air for 3.5 days, indicating that the Type 3 centers remain reduced. In contrast, both the Type 2 and Type 3 centers in fully reduced TlHg Lc completely reoxidize rapidly upon exposure to air. Taken together, these results indicate that the Lc Type $2 \mathrm{Cu}$ is critically required for facile reactivity with $\mathrm{O}_{2}$, whereas the Type 1 center is unnecessary. Thus, the Type 2-Type 3 trinuclear $\mathrm{Cu}$ cluster represents the minimum functional unit required for $\mathrm{O}_{2}$ reactivity.

\section{THE TRINUCLEAR CU CLUSTER}

Having defined the reactivity of the trinuclear $\mathrm{Cu}$ cluster, we have employed a combination of abs., CD and LTMCD spectroscopies to characterize the electronic and geometric structure of the trinuclear Type 2-Type $3 \mathrm{Cu}$ cluster and to probe its small molecule interactions. In order for an electronic transition to be observed in the LTMCD spectrum, it should have significant absorption intensity and be associated with a degenerate ground state (C-term $M C D$ ). CT and $d \rightarrow d$ transitions at the paramagnetic $(S=1 / 2)$ Type $2 \mathrm{Cu}$ (II) will thus be strong in the LTMCD spectrum. In contrast, the pair of $\mathrm{Cu(II)}$ ions in the Type 3 center are antiferromagnetically coupled, giving rise to an $S=0$ ground state. Thus, transitions of the Type 3 center will only be observed in abs. and $C D$.

The Type $2 \mathrm{~d} \rightarrow \mathrm{d}$ bands are evident in the LTMCD of T1Hg Lc (Fig.4), and their energies define a tetragonal effective geometry at this site. Three sharp CT bands are present at 320 -

$370 \mathrm{~nm}$, indicating that the Type 2 center exhibits significant contribution in this spectral region. Fluoride is known to bind with very high affinity to the Type $2 \mathrm{Cu}(\mathrm{II})\left(\mathrm{K}>10^{4} \mathrm{M}^{-1}\right)$. 
Addition of $F^{-}$causes a dramatic decrease in the amplitude of the $d \rightarrow d$ and CT MCD features, thus confirming their assignment to the Type 2 site. A band at $-900 \mathrm{~nm}$, which is observed in the CD but not LTMCD spectra, is assigned as a $d \rightarrow d$ band of the diamagnetic Type 3 site. Upon binding $\mathrm{F}$ - at the Type $2 \mathrm{Cu}$, the Type $3 \mathrm{~d} \rightarrow \mathrm{d}$ band shifts to lower energy, indicating an additional strong interaction of this exogenous ligand with the Type 3 site.

The mechanism of $\mathrm{N}_{3}{ }^{-}$binding to TlHg Lc has been probed at the molecular level via LTMCD and abs. studies of $\mathrm{N}_{3}^{-} \rightarrow \mathrm{Cu}$ (II) CT features. Upon binding $\mathrm{N}_{3}^{-}$, a paramagnetic $\mathrm{N}_{3} \rightarrow$ Type 2 CT band at $485 \mathrm{~nm}$ and a perturbation of the Type $3 \mathrm{~d} \rightarrow \mathrm{d}$ transition titrate in parallel (K $\sim 700$ $\mathrm{M}^{-1}$ ). In addition, this $\mathrm{N}_{3}^{-}$uncouples $<10 \%$ of the diamagnetic Type 3 sites, rendering them paramagnetic, and giving rise to three paramagnetic $\mathrm{N}_{3}{ }^{-} \rightarrow$ uncoupled Type 3 CT bands, and a broad triplet EPR signal. Thus, a single azide bridges the Type 2 and Type 3 centers (Fig. $5)$. The similarity of the $\mathrm{N}_{3}{ }^{-}$-induced uncoupling process and Type $3 \mathrm{~d} \rightarrow \mathrm{d}$ perturbations in $\mathrm{T} 2 \mathrm{D}$ and $\mathrm{T} 1 \mathrm{Hg} \mathrm{Lc}$ indicate that the interaction of $\mathrm{N}_{3}{ }^{-}$with the Type 3 center is not significantly perturbed by interaction with the Type 2 center.

Thus, we have determined that the Type 2 and Type 3 centers in the multicopper oxidases comprise a trinuclear $\mathrm{Cu}$ cluster capable of exogenous ligand bridging, and that this represents the active site for the multielectron reduction of $\mathrm{O}_{2}$. Detailed studies of the molecular mechanism of this reaction are currently underway.

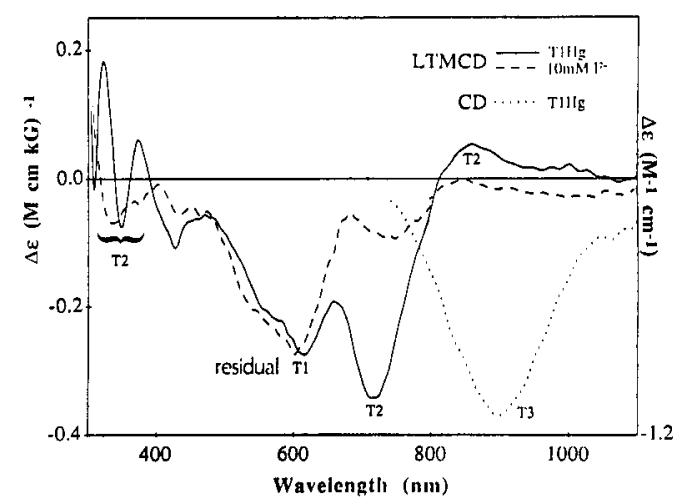

Fig 4. Comparison of the LTMCD and CD spectra of T1Hg laccase.

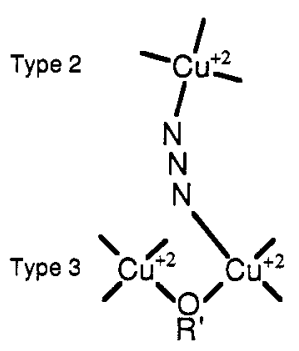

Fig. 5. Model for bridged binding of $\mathrm{N}_{3}{ }^{-}$ at the trinuclear $\mathrm{Cu}$ cluster.

\section{Acknowledgements}

E.I.S. would like to thank the students, postdoctoral associates, and collaborators who have made major contributions to this research. NIH (grant \#DK31450) is gratefully acknowledged for supporting this research.

\section{REFERENCES}

1. N.C. Eickman, R.S. Himmelwright and E.I. Solomon, Proc. Nat1. Acad. Sci. USA 76, 2094 (1979).

2. R.S. Himmelwright, N.C. Eickman, C.D. LuBien, K. Lerch and E.I. Solomon, J. Am. Chem. Soc, 102,7339 (1980).

3. W.P.J. Gaykema, W.G.J. Hol, J.M. Verijken, N.M. Soeter, H.J. Bak and J.J. Beintema, Nature 309,23 (1984).

4. M.E. Winkler, K. Lerch and E.I. Solomon, J. Am. Chem. Soc, 103, 7001 (1981).

5. D.E. Wilcox, A.G. Porras, Y.T. Hwang, K. Lerch, M.E. Winkler and E.I. Solomon, J. Am. Chem. Soc. 107, 4015 (1985).

6. D.J. Spira and E.I. Solomon, J.Am. Chem. Soc. 109, 6421 (1987).

7. (a) M.D. Allendorf, D.J. Spira and E.I. Solomon, Proc. Nat1. Acad. Sci. USA 82,3063 (1985). (b) D.J. Spira, M.D. Allendorf and E.I. Solomon, J. Am. Chem. Soc. 108, 5319 (1986).

8. A. Messerschmidt, A. Rossi, R. Ladenstein, R. Huber, M. Bolognesi, R. Petruzelli and A. Finazzi-Agro, In Oxidases and Related Systems, T.E. King, H.S. Mason and M. Morrison, Eds., p. 285, Alan R. Liss, New York (1988).

9. J.E. Pate, R.W. Cruse, K.D. Karlin and E.I. Solomon, J.Am, Chem. Soc, 109, 2624 (1987).

10. J.E. Pate, P.K. Ross, T.J. Thamann, C.A. Reed, K.D. Karlin, T.N. Sorre11 and E.I. Solomon, J. Am. Chem. Soc, in press (1989).

11. T.D. Westmoreland, D.E. Wilcox, M.J. Baldwin, W.B. Mims and E.I. Solomon, J. Am. Chem. Soc. in press (1989).

12. L.-S. Kau, D.J. Spira-Solomon, J.E. Penner-Hahn, K.O. Hodgson and E.I. Solomon, J. Am. Chem. Soc. 109, 6433 (1987). 